\title{
IgA Nephropathy: A Chinese Perspective
}

\author{
Zhao Zhang a, b, c, d Yuemiao Zhang a, b, c, d Hong Zhang a,b, c, d \\ aRenal Division, Department of Medicine, Peking University First Hospital, Beijing, China; ${ }^{b}$ Institute of Nephrology, Peking

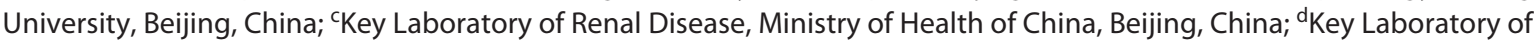 \\ Chronic Kidney Disease Prevention and Treatment, Peking University, Ministry of Education, Beijing, China
}

\section{Keywords}

Genetics · Glomerulonephritis · IgA nephropathy ·

Pathogenesis · Treatment

\section{Abstract}

Background: IgA nephropathy ( $\lg \mathrm{AN}$ ) is the most common primary glomerular disease worldwide and remains a leading cause of chronic kidney disease and end-stage renal disease. The disease prevalence, clinical and pathological phenotypes, the underlying pathogenic molecular mechanisms, and the response to treatments are highly heterogeneous in different ethnic populations, which raise the concern that IgAN may differ across different parts of the world. Summary: From a Chinese perspective, we stated the disease burden of IgAN, summarized genome-wide association studies and research into pathological molecules, and compared them with findings based on other populations. The emerging biomarkers, indigenous clinical trials, and major challenges for Chinese researchers and nephrologists in studying IgAN are also discussed. Key Messages: In this review, we described a higher risk of major susceptible loci in mucosal immunity, IgA production, and complement activation pathways in Chinese patients with IgAN. With our understanding of the pathogenesis of IgAN, novel biomarkers are emerging. Although there are challenges for conducting high-quality clinical trials in China, it is still feasible to conduct innovative and well-designed studies of IgAN. In the future, international collaborations on research infrastructure would be helpful to advance clinical and basic research in China.

(C) 2021 The Author(s)

Published by S. Karger AG, Basel

\section{Introduction}

IgA nephropathy (IgAN) is the most common primary glomerular disease worldwide and remains a leading cause of chronic kidney disease and end-stage renal disease (ESRD) [1-3]. Diagnosis of IgAN is established by the presence of immunoglobulin A1 (IgA1) as the dominant or codominant immunoglobulin in the glomerular mesangium on examination of renal biopsy [4-7]. Although the exact pathogenesis of IgAN remains to be determined, a multi-hit theory has been proposed [8]. The fundamental steps are the generation of galactose-deficient IgA1 (Gd-IgA1); antibody formation against Gd-

Zhao Zhang and Yuemiao Zhang contributed equally to this work. karger@karger.com www.karger.com/gdz

Karger $\stackrel{\text { ' }}{=}$
(C) 2021 The Author(s)

Published by S. Karger AG, Basel

This is an Open Access article licensed under the Creative Commons Attribution-NonCommercial-4.0 International License (CC BY-NC) (http://www.karger.com/Services/OpenAccessLicense), applicable to the online version of the article only. Usage and distribution for commercial purposes requires written permission.
Correspondence to:

Hong Zhang, hongzh@bjmu.edu.cn 
IgA1; immune complex formation and mesangial deposition; and complement activation. This culminates in macrophage infiltration and tubulointerstitial inflammation, leading to renal damage. However, there are substantial variations in the prevalence and phenotype of IgAN globally, which might reflect a complex, yet poorly understood, interaction between genetic and environmental factors that modulate the disease phenotype in individuals and across different populations. Notably, there is a clear West-to-East prevalence gradient, with the disease being most common in China (45-58\% of primary glomerulonephritis) and other Asian countries (47\% in Japan, 28\% in Korea, and 45\% in Singapore), compared with that in European countries (10-35\% of primary GN) [9-16]. Generally, the disease affects males more frequently than females, with a male-to-female ratio as high as 3:1 in Europeans [17]; however, this ratio approximates to $1: 1$ in China, a pattern that might be shaped by differences in genetics or local environments $[18,19]$. These differences might reflect differences in examination or biopsy policies among populations. For instance, systematic mass screening of urine is more common in some Asian regions (Hong Kong, Japan, Korea, and Singapore) than in Western countries. Persistent microscopic hematuria and/or mild proteinuria in apparently healthy individuals might not be recognized as possible IgAN, and therefore IgAN might be underestimated in Western countries. Furthermore, different indications for kidney biopsy are adopted by nephrologists in individuals with persistent urinary abnormalities [20]. Finally, the ethnic heterogeneity of IgAN might also result from a true geographical variation in the disease.

China's population exceeds 1.4 billion people and as a nation, it has among the highest prevalence of IgAN. This results in a heavy disease burden. Importantly, recent evidence from genetic, cell biology, and clinical studies has provided us with a chance to better understand IgAN from a Chinese perspective, which will advance our understanding and improve therapy in a more specific way.

\section{Disease Burden of IgAN in China}

Although in most cases IgAN is a chronically progressive disease and is not life-threatening, it is nevertheless important to develop effective treatment, especially because it often affects young people, in whom a long and progressive disease course might lead to ESRD. A high frequency of IgAN has been observed in children and junior high school students, both in clinical practice and in

IgA Nephropathy from a Chinese

Perspective nationwide mandatory mass urine screening studies in Chinese, Japanese, and Korean schools [21, 22]. Moreover, 1 study analyzed 34,630 hospitalized patients undergoing native renal biopsy in China retrospectively and found that IgAN (24.09\%) was the most common primary glomerulonephritis [23]. When the number of renal biopsies increased, the percentage of IgAN diagnoses also increased. Many retrospective studies have shown that $30-50 \%$ of biopsy-proven patients with IgAN develop ESRD in approximately 20 years after diagnosis [24, 25]. The increasing incidence of ESRD has a major impact on the need for dialysis, including maintenance hemodialysis and peritoneal dialysis [26]. However, not all patients have access to renal replacement treatment because of the high cost of treatment [26], which places a large economic burden on individuals and health care resources. Given the high prevalence and its associated morbidity and cost, IgAN has become a major public health problem for the Chinese government [27]. Thus, there is an urgent need to understand the underlying pathogenic mechanism of IgAN, especially from a Chinese perspective.

\section{Pathogenesis of IgAN from a Chinese Perspective}

\section{Genetic Studies}

Genome-wide association studies (GWASs) have revolutionized our capacity to identify genetic susceptibility loci in many diseases, including IgAN. To date, 6 large GWASs have been performed in European populations (France, Italy, UK, and the USA) and/or Chinese populations with IgAN [28-33]. Overall, 21 loci were detected (Table 1). Most of the detected susceptibility loci were shared between European and Chinese populations, and are involved in the regulation of mucosal immunity (VAV3, DEFA, and CARD9), IgA production (ST6GAL1, TNFSF13, and HORMAD2), antigen presentation and adaptive immunity $(M H C)$, and complement activation (CFH, CFHR3-1, and ITGAM-ITGAX) pathways [34-36], which were largely consistent with the multi-hit pathogenesis model of IgAN. The risk allele frequencies and odds ratios of the genes involved in antigen presentation (MHC), mucosal immunity (TNFSF13 and HORMAD2), leukocyte-specific complement receptor formation (ITGAM-ITGAX), and alternative complement pathway activation (CFH and CFHR3-1) tend to be higher and larger in Han Chinese populations than in European populations. Accordingly, more severe clinical presentation and higher risk of disease progression, as well as active lesions, such as mesangial hypercellularity and crescents [37, 38], 
Table 1. Susceptibility loci of IgAN discovered in GWASs

\begin{tabular}{|c|c|c|c|c|c|c|c|c|c|}
\hline Locus & SNP & RA & Gene & \multicolumn{4}{|c|}{ Asians } & \multicolumn{2}{|c|}{ Europeans } \\
\hline $1 p 36.13$ & rs2240335 & $A$ & PADI4 & 0.60 & 1.18 & $7.07 \mathrm{E}-03$ & 0.41 & 1.16 & $1.54 \mathrm{E}-02$ \\
\hline $1 \mathrm{q} 23.1$ & rs6427389 & $\mathrm{C}$ & FCRL3 & 0.79 & 1.13 & $1.01 \mathrm{E}-01$ & 0.66 & 1.05 & $3.96 \mathrm{E}-01$ \\
\hline $1 q 32$ & rs6677604 & G & CFHR3,1-del & 0.93 & 1.57 & $6.70 \mathrm{E}-07$ & 0.8 & 1.3 & $2.40 \mathrm{E}-09$ \\
\hline \multirow{6}{*}{$6 p 21$} & rs2856717 & G & & 0.77 & 1.38 & $5.00 \mathrm{E}-10$ & 0.62 & 1.21 & $4.70 \mathrm{E}-08$ \\
\hline & rs2523946 & $\mathrm{C}$ & HLA-A & 0.54 & 1.21 & $1.74 \mathrm{E}-11$ & & & \\
\hline & rs660895 & G & HLA-DRB1 & 0.28 & 1.34 & 4.13E-20 & & & \\
\hline & rs1794275 & $A$ & HLA-DQA/B & 0.19 & 1.30 & $3.43 \mathrm{E}-13$ & & & \\
\hline & rs2071543 & G & TAP2/PSMB9 & - & 1.41 & $2.10 \mathrm{E}-09$ & - & 0.99 & $8.50 \mathrm{E}-01$ \\
\hline & rs9275596 & $\mathrm{T}$ & HLA-DR/DQ & 0.80 & 1.69 & $1.10 \mathrm{E}-19$ & 0.65 & 1.35 & $1.60 \mathrm{E}-15$ \\
\hline \multirow{4}{*}{$8 p 23$} & rs10086568 & A & & 0.27 & 1.2 & $1.10 \mathrm{E}-05$ & 0.33 & 1.14 & $1.60 \mathrm{E}-05$ \\
\hline & rs2738058 & $\mathrm{T}$ & & 0.73 & 1.31 & $5.75 \mathrm{E}-10$ & & & \\
\hline & rs9314614 & $\mathrm{C}$ & & 0.40 & 1.19 & $3.54 \mathrm{E}-05$ & & & \\
\hline & rs12716641 & $\mathrm{T}$ & & 0.78 & 1.26 & $7.80 \mathrm{E}-07$ & & & \\
\hline $9 q 34$ & rs4077515 & $\mathrm{T}$ & CARD9 & 0.28 & 1.11 & $8.00 \mathrm{E}-03$ & 0.4 & 1.18 & $2.10 \mathrm{E}-08$ \\
\hline \multirow[t]{3}{*}{$16 p 11.2$} & rs11150612 & A & ITGAM-ITGAX & 0.75 & 1.17 & $2.50 \mathrm{E}-04$ & 0.36 & 1.19 & $1.20 \mathrm{E}-08$ \\
\hline & rs11574637 & $\mathrm{T}$ & & 1.00 & - & - & 0.82 & 1.32 & $8.10 \mathrm{E}-13$ \\
\hline & rs7190997 & $\mathrm{C}$ & & 0.72 & 1.21 & $4.00 \mathrm{E}-05$ & & & \\
\hline \multirow[t]{2}{*}{$17 p 13.1$} & rs3803800 & $A$ & TNFSF13 & 0.32 & 1.17 & $4.60 \mathrm{E}-05$ & 0.2 & 1.08 & $2.50 \mathrm{E}-02$ \\
\hline & rs4227 & G & & 0.24 & 1.23 & $4.31 \mathrm{E}-10$ & & & \\
\hline $22 q 12$ & rs2412971 & G & $\begin{array}{l}\text { HORMAD2, MTMR3, LIF, } \\
\text { OSM }\end{array}$ & 0.67 & 1.35 & $6.00 \mathrm{E}-11$ & 0.54 & 1.13 & $1.50 \mathrm{E}-04$ \\
\hline \multicolumn{10}{|l|}{ Han Chinese } \\
\hline
\end{tabular}

Note: Genes with higher risk allele frequency or larger odds ratios in Asian/Chinese populations were highlighted in bold. SNP, single nucleotide polymorphism; RA, risk allele; RAF, risk allele frequency; OR, odds ratio; IgAN, IgA nephropathy; GWASs, genome-wide association studies; CFHR, complement factor H-related protein.

have been reported in Chinese patients with IgAN than in European patients. More interestingly, the relative importance of each "hit" might vary in different ethnic populations, providing genetic insights into more targeted treatment in China. In the following sections, we will focus more specifically on the well-studied mucosal immunity, Gd-IgA1 production, and complement activation.

\section{Mucosal Immunity}

IgA production and abnormalities in circulating IgA are the initial events in IgAN pathogenesis [8]. The de- posited IgA and the ensuing response of the mesangium are crucial to IgAN development [2]. The source of this pathogenic IgA in IgAN is an emerging area of study. Both mesangial and serum IgA are polymeric, and because polymeric IgA is normally produced at mucosal surfaces, this suggests that IgAN is intimately linked with abnormal mucosal immune responses to microorganisms [2]. In addition, novel associations at ST6GAL1 on chromosome 3q27.3, ACCS on chromosome 11p11.2, and ODF1-KLF10 on chromosome 8q22.3 were identified in a Chinese population [39]. Most of these loci implicate
32

Glomerular Dis 2022;2:30-41 DOI: $10.1159 / 000520039$ 
genes involved in mucosal immunity in the gut and IgA production [39]. These findings supported the concept that mucosal immunity might play a fundamental role in the pathogenesis of IgAN because hematuria is one of the typical clinical features of the disease and gross hematuria is frequently seen after mucosal infections [40]. The immune response activated against mucosal antigen exposure to bacterial, viral, or alimentary components can lead to the activation of dendritic cells, T cells, and B cells, and eventually, the production of IgA. Therefore, attempts to modulate the mucosal immune system would be beneficial for patients with IgAN.

As suggested, most of the identified mucosal-related loci fulfill the immunological function not only of gutassociated lymphoid tissue (GALT), but also nasopharyngeal-associated lymphoid tissues (NALT)/bronchialassociated lymphoid tissues. Using the gddY mouse model of spontaneous IgAN, NALT was suggested to be the major induction site of IgAN [41]. Accordingly, tonsillectomy has been shown to improve urinary findings and reduce IgA deposits in patients from China and Japan, but this was not observed in patients from European countries [42, 43]. By contrast, in a recent clinical survey on the presentation and clinical management of patients with IgAN in Europe and Japan, gastrointestinal complications, including inflammatory bowel diseases and celiac disease, were more frequent in European patients than in Japanese patients [44]. Such findings are compatible with the clinical trial NEFIGAN, in which targeted release of budesonide in the distal ileum was reported to reduce proteinuria and stabilize renal function in patients with IgAN [45]. Further exploration of the molecular mechanisms of GALT, NALT, and bronchial-associated lymphoid tissues, as well as the immune crosstalk between them, would provide more insights into the targeting mucosal therapy in different ethnic groups.

\section{Gd-IgA1 Levels}

Gd-IgA1 plays an essential role in the postulated pathogenesis of IgAN [8]. Elevated levels of Gd-IgA1 have been reported in patients with IgAN of Caucasian [46, 47], Asian [48, 49], and African ancestry [50]. A higher Gd-IgA1 quartile was associated with the progression of renal disease in a large cohort of Chinese patients with IgAN [49]. However, variations identified in C1GALT1C1 and $C 1 G A L T 1$ explain approximately $7 \%$ of the variability in circulating Gd-IgA1 in European patients, but only $2 \%$ in Chinese patients $[51,52]$. A recent GWAS of GdIgA1 in China identified 2 new variants, GALNT12 and C1GALT1 [53]. Moreover, despite the higher incidence

IgA Nephropathy from a Chinese Perspective and poorer prognosis of IgAN in China, the C1GALT1 risk haplotype is found 10 times less frequently in Chinese patients than in European patients [54], suggesting heterogeneity of the pathogenic role of Gd-IgA1 between Caucasian and Chinese patients with IgAN. Actually, there is a wide distribution in the proportion of $\operatorname{IgA} 1 \mathrm{O}$ glycoforms in the serum, and there is significant overlap in the levels of these IgA $1 \mathrm{O}$-glycoforms between patients with IgAN and healthy individuals. Moreover, levels of Gd-IgA1 in Chinese cases of IgAN were lower than in Caucasian cases and were even comparable with those seen in a healthy Caucasian population [55]. The overlap in the levels of Gd-IgA1 between patients with IgAN and healthy controls, as well as the comparable levels of Gd-IgA1 between Chinese patients with IgAN and healthy controls in Europe raise important and as yet unanswered questions concerning the pathogenic importance of changes in IgA1 O-galactosylation in different ethnic populations. Besides the quantity of $\mathrm{Gd}-\mathrm{IgA} 1$, host genetic susceptibility and different sites of Gd-IgA1 [56] might also play an important role, representing features that deserve additional study.

\section{Complement Activation}

Complement proteins are activated in IgAN. Complement component C3 frequently colocalizes with IgA, while $\mathrm{Clq}$ is absent, suggesting that activation of complement mainly occurs via the alternative pathway or lectin pathway in IgAN. C3 is the most abundant complement component, found in up to $90 \%$ of cases in renal biopsy specimens $[57,58]$. Apart from C3, other complement elements can also be codeposited with IgA. More recently, mass-spectrometry analysis of micro-dissected glomeruli from IgAN kidney biopsy specimens showed significant amounts of alternative-pathway regulation proteins, such as factor $\mathrm{H}$, and complement factor $\mathrm{H}$-related proteins (CFHRs) 1, 2, 3, and 5 [59]. Clinically, a Chinese study of 202 patients with IgAN showed that urinary factor $\mathrm{H}$ levels were significantly higher in patients with IgAN than in healthy controls, and were also associated with severe histological findings [60]. Concurrently, another study from China, including 1,126 patients with IgAN and regular follow-up and 153 unrelated healthy individuals, demonstrated that circulating CFHR5 levels were elevated significantly in patients with IgAN and were associated with a lower estimated glomerular filtration rate (eGFR), hypertension, and severe Oxford-T and Oxford-C scores [61]. Genetically, GWAS data showed that a common combined deletion of CFHR 1 and CFHR3 has a protective function in IgAN in a Han Chinese pop- 
Table 2. Prediction models of IgAN

\begin{tabular}{|c|c|c|c|c|c|c|}
\hline Japanese & 790 & $\begin{array}{l}\text { Proteinuria, serum albumin, hematuria, diastolic } \\
\text { blood pressure, total protein }\end{array}$ & Customized scoring & - & Yes [86] & {$[70,71]$} \\
\hline Chinese & 1,025 & $\begin{array}{l}\text { Age, serum albumin, hypertension, serum uric } \\
\text { acid, hematuria, urinary protein, serum creatinine }\end{array}$ & $\begin{array}{l}\text { Oxford classification score } \\
(\mathrm{M}, \mathrm{S}, \mathrm{T})\end{array}$ & 0.89 & No & {$[72]$} \\
\hline Caucasian & 332 & Blood pressure, proteinuria & Customized scoring & - & Yes [87] & {$[74]$} \\
\hline Multiple ethnic & $=3,927$ & $\begin{array}{l}\text { eGFR, blood pressure, and proteinuria at biopsy; } \\
\text { kidney biopsy (MESTC), age, medication use, race }\end{array}$ & Customized scoring & $\begin{array}{l}0.82 \text { (with } \\
\text { race) }\end{array}$ & No & {$[75]$} \\
\hline Chinese & 934 & Sex, age, eGFR, hemoglobin, proteinuria & Oxford classification score $(\mathrm{M}, \mathrm{T})$ & 0.86 & No & {$[88]$} \\
\hline
\end{tabular}

ulation [62]. In addition, it was reported that rare variants of CFHR5 contribute to genetic susceptibility to IgAN [61]. Moreover, the presence of mannan-binding lectin (MBL) with IgA, C3, and C4d is consistent with activation of the lectin pathway in some patients [1]. MBL deposits, found in about $25-35 \%$ of patients, have been associated with higher proteinuria, lower eGFR, and more severe histopathological lesions $[63,64]$. In a Chinese study of 162 patients with IgAN, urinary MBL levels were associated significantly with impaired renal function and increased proteinuria [65]. A study that measured MBL2 variants and MBL levels in 749 patients with IgAN and 489 healthy controls from a Chinese cohort also found that patients with IgAN and MBL deficiency had a higher incidence of prodromic infections and gross hematuria compared with those with sufficient MBL levels (100$3,540 \mathrm{ng} / \mathrm{mL}$ ) and that patients with high MBL levels $(>3,540 \mathrm{ng} / \mathrm{mL})$ had more severe proteinuria and a higher proportion of crescents, which suggested that MBL contributes to IgAN pathogenesis through multiple mechanisms [66]. These observations have generated tremendous interest in the important role of complement activation in Chinese patients with IgAN, and targeting complement pathways could be a promising treatment approach.

Collectively, the identification of IgAN susceptibility loci from GWASs has greatly advanced our understanding of the disease pathogenesis of IgAN. The identification of susceptibility loci shared between different ethnic populations, as well as those detected in the Chinese population, suggests that the relative importance of each "hit," such as Gd-IgA1 production and complement acti- vation, might vary in different ethnic populations. In the future, a better understanding of the pathogenic pathways operating in different ethnic populations and the discovery of better biomarkers might help to develop more precise targeted treatment for IgAN.

\section{Risk Prediction and Monitoring of IgAN in China}

IgAN is characterized by various clinical and histological manifestations, and some patients with high risk show a progressive disease course. Identifying the risk of progression early in the course of the disease remains problematic. Several well-established clinicopathogenic variables, including eGFR, proteinuria, blood pressure, body mass index, and Oxford classification scores, have been reported to be associated with disease progression [67-69]. Using these clinicopathogenic variables, several risk-prediction models have been developed (Table 2). Although their discrimination performance is well accepted (C statistics $>0.85$ ), only a few of them were confirmed using external validation and were able to identify high-risk patients accurately [70-74]. To account for ethnic differences, Barbour et al. [75] developed prediction models that were shown to be accurate and valid methods to predict disease progression and patient risk stratification in IgAN in multiethnic cohorts $[75,76]$. An updated tool for use in children has been reported [77]. However, in an independent external validation study in China, the risk probability over 3 years was shown to be overestimated [78], suggesting the necessity to develop prediction models suitable for Chinese patients with
Zhang/Zhang/Zhang 
Fig. 1. Process for the identification of eligible clinical trials in IgAN in China. Results of a systematic search on IgAN in China using ClinicalTrials.gov (https://clinicaltrials.gov/). IgAN, IgA nephropathy.

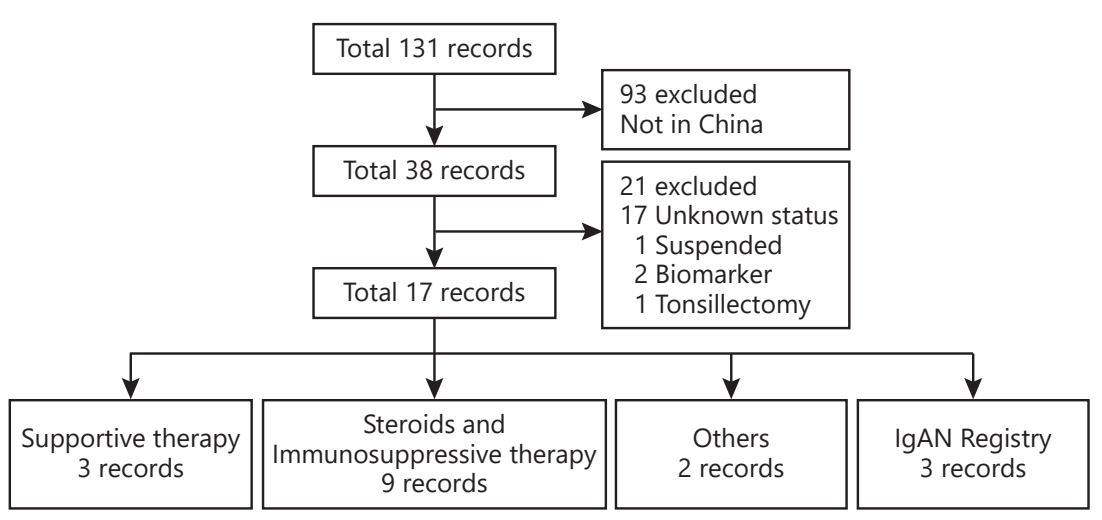

grates genomic data generated from next-generation sequencing, whole-exome sequencing, and whole-genome sequencing, into risk scores for prognostication and treatment decision-making has been attempted in European patients (IgAN-Genetic Risk Score, IgAN-GRS). Moreover, using updated proteomic or metabolomic methods, noninvasive biomarkers from blood or urine for the early diagnosis of IgAN are emerging rapidly.

Collectively, a wide range of studies have been conducted and various biomarkers have been introduced. Considering the heavy disease burden and the significant heterogeneity of IgAN in China, greater efforts should be made to develop risk-prediction models by combining these biomarkers, which would help to predict the risk of renal failure and identify patients who might benefit from more aggressive therapy.

\section{Clinical Trials of IgAN in China}

Our increased understanding of the pathogenesis of IgAN and racial differences, and the large number of patients with IgAN in China, provide a great potential to conduct clinical trials and seek precision therapies. We summarized the registered clinical trials of IgAN in China through searching ClinicalTrials.gov (Fig. 1). Overall, 17 trials (including 3 IgAN registries) of IgAN were conducted by investigators in China. However, as shown in Table 3, most of these trials tend to be limited by relatively small numbers of patients or their singlecenter nature, and few of them were cited in the KDIGO guidelines of glomerular diseases. Notably, despite challenges for high-quality clinical trials, it is feasible to perform innovative and well-designed studies in China. 


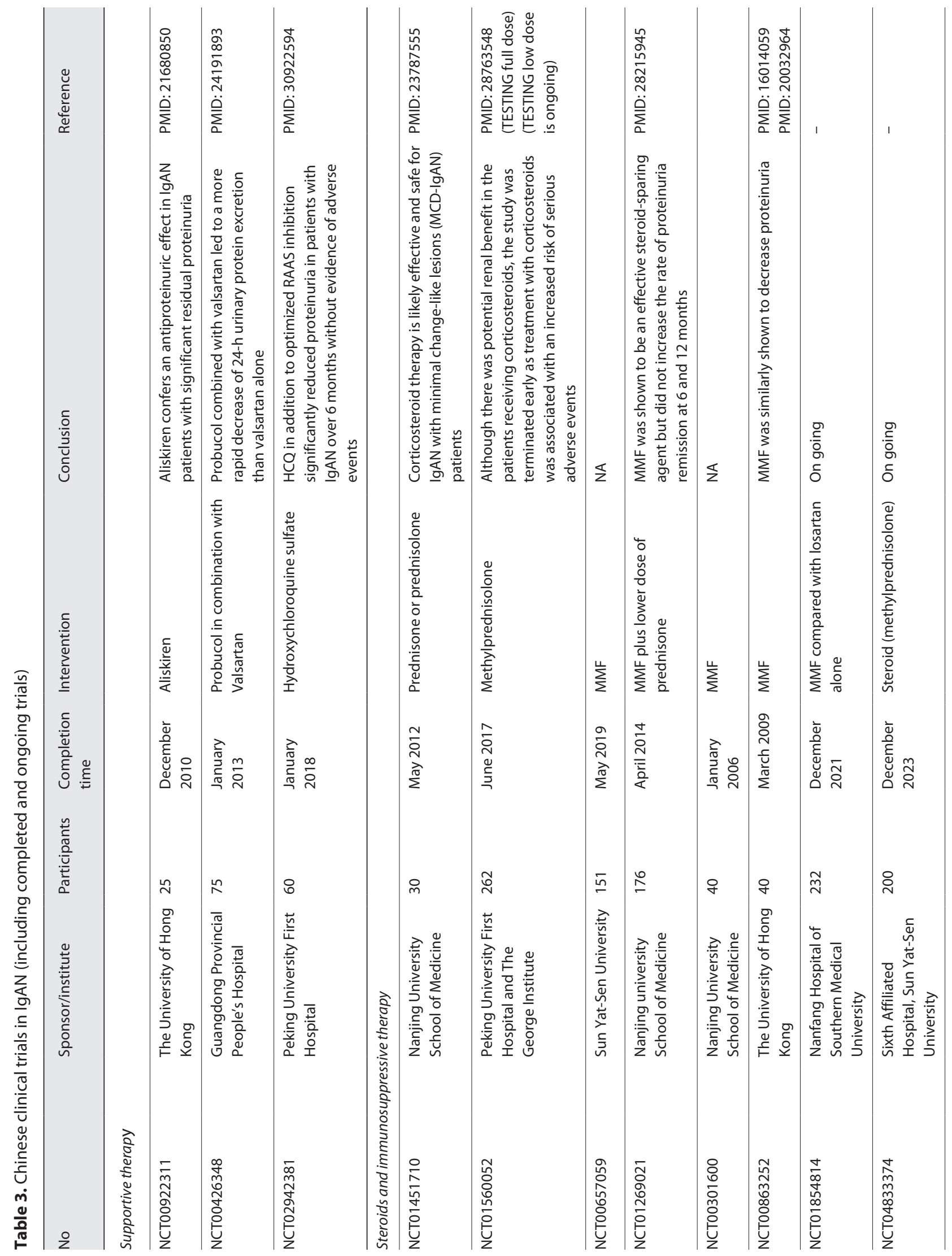

36 


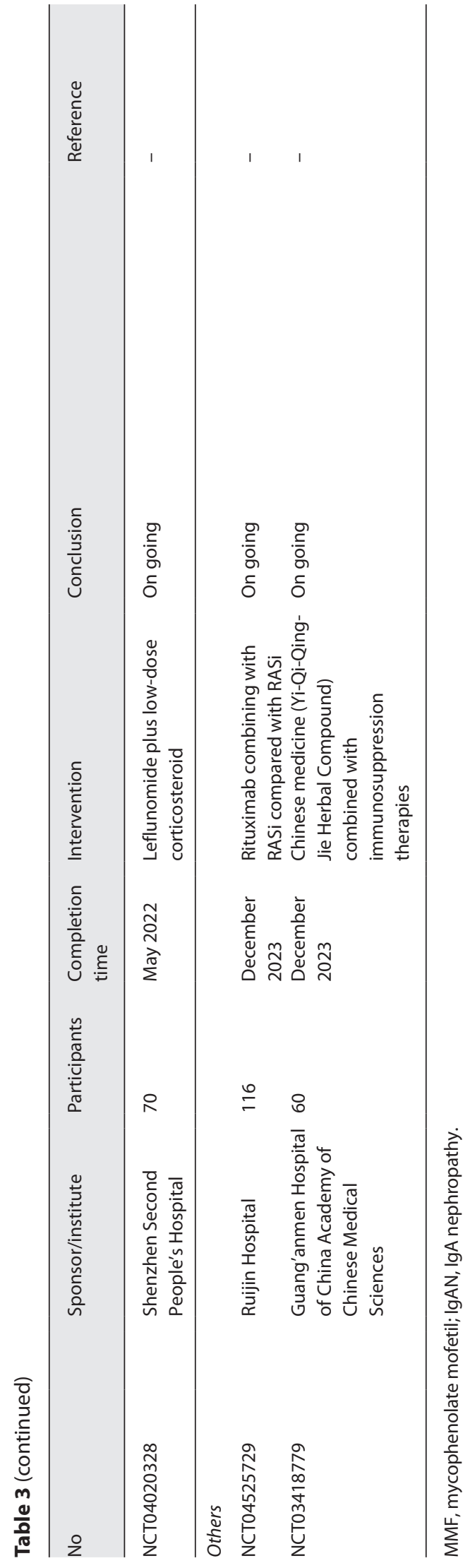

IgA Nephropathy from a Chinese Perspective
The TESTING study (NCT01560052) is an international clinical trial designed to evaluate the long-term efficacy and safety of oral methylprednisolone on a background of RAS inhibitor therapy in patients with IgAN at a high risk of progression. The TESTING full dose study, including 262 patients from 67 locations globally (majority Chinese) with proteinuria $>1 \mathrm{~g} /$ day and eGFR $20-120 \mathrm{~mL} / \mathrm{min}$ per $1.73 \mathrm{~m}^{2}$, randomized to corticosteroids or placebo, demonstrated a potential renal benefit in the patients receiving corticosteroids [81], although the study was terminated early because treatment with corticosteroids was associated with an increased risk of adverse events [81]. Interestingly, the major outcome of the TESTING study was different from the STOP-IgAN study, which included 162 European patients with proteinuria $>0.75 \mathrm{~g} /$ day randomized to optimal supportive care, with or without immunosuppression [82]. The STOP-IgAN study showed that the addition of immunosuppressive therapy to optimal supportive care did not change the rate of eGFR loss [82]. The difference in the ability to demonstrate benefit from immunosuppressive therapy in IgAN in TESTING versus STOPIgAN is most likely explained by differences in patient characteristics. Patients in the TESTING study (majority Chinese) appeared to show more aggressive disease progression, as seen in the higher baseline proteinuria levels and more rapid rates of eGFR decline. Previously, in Chinese patients with IgAN with higher risk, mycophenolate mofetil (MMF) treatment was shown to be effective. In a Chinese clinical trial, 176 patients with IgAN with active proliferative lesions were randomized to MMF with low-dose steroids versus steroids alone. MMF was shown to be an effective corticosteroid-sparing agent in proteinuria remission at 6 and 12 months (NCT01269021) [83]; however, MMF efficacy was not observed in a non-Chinese IgAN population. Therefore, the successful experiences from the above-mentioned clinical trials highlight the importance of the "right population" and the "right time" for treatment. In the future, designing and conducting high-quality clinical trials, especially those with precision medicine designs, and clinical trials stratified by clinical or pathological phenotypes, might be more informative for Chinese patients, based on the high heterogeneity and more severe clinical and pathological phenotypes in Chinese patients with IgAN. Besides clinical trials conducted by independent investigators, Chinese nephrologists also actively participate in novel international clinical trials in IgAN, including those assessing therapies targeting specific targets in disease pathogenesis, such as the 
complement Factor B Inhibitor-LNP023 [84], the MASP-2 Inhibitor-OMS721 (Narsoplimab) [85], and the targeted-release budesonide-Nefecon (45), among others. Pragmatic clinical studies based on real-world experience might offer an important option to Chinese patients with IgAN and investigators. Three upcoming IgAN databases are registered in China. One is the IgA Nephropathy Registration Initiative of High Quality (NCT03001947), which will recruit 10,000 participants with a 10-year follow-up duration and should be completed before 2026. Another is the Chinese Registry of Prognostic Study of IgA Nephropathy (NCT04858724), which will recruit 2,000 participants with a 12 -month follow-up duration and will be completed before 2030 . The last 1 is the Registry of IgA Nephropathy in Chinese Children (RACC) (NCT03015974), which aims to identify a safe and effective treatment option for IgAN in children and will perform prospective registration among 25 pediatric nephrology medical centers in China with 1,200 participants and a 24-month follow-up duration.

\section{Challenges and Future Directions}

Advances in genetic, basic, experimental, and clinical studies have greatly improved our understanding of the pathogenesis of IgAN and have provided us with potential novel biomarkers and therapeutic strategies. However, explaining the heterogeneity of IgAN remains a challenge.

First, a wide spectrum of clinical and pathological features of IgAN has been observed, implying that IgAN might not be the same disease across populations, or even within the same ethnic group. It is truly challenging that the diagnosis of IgAN mainly depends on renal biopsy with a demonstration of predominant $\operatorname{IgA} 1$ deposition in the glomerular mesangium. With a more in-depth understanding of IgAN pathogenesis, clearer diagnostic criteria could be expected in the future.

Second, the impact of confounding environmental factors and the difficulty of movement of human tissue across borders mean that we can never be sure if a difference across populations is simply the result of a difference in laboratory methodologies. In the future, trans-ethnic genetic studies or multicenter-based basic research collaborations are needed to truly compare the differences between populations.

Third, the current trials in developed countries might not benefit patients in China because of a distinct etiol- ogy. Although a culture of appreciation for high-quality clinical trials is developing in China, the lack of appropriate infrastructure, weak administrative capacity, and limited sources of funding make the performance of high-quality clinical trials challenging. In the future, collaborations like the IgA Nephropathy Registration Initiative of High Quality and the Chinese Registry of Prognostic Study of IgA Nephropathy could facilitate the conduct of large cohort and multicenter-based clinical trials to test the efficacy of novel therapies for patients with IgAN in China. In addition, international academic collaborations bringing the rigor of design and execution of multicenter trials will represent a most welcome evolution in the landscape of clinical trials for IgAN. In this regard, studies like the UK IgAN RaDaR cohort, which was expanded internationally using a web-based registry collecting standardized data and standardized samples, and which protocolized the monitoring and follow-up of patients with IgAN, have the potential to drive global studies of IgAN. This will allow systematic comparisons of the disease across ethnicities to understand variations in the susceptibility and severity of the disease in specific groups that traditionally have only been studied in isolation.

\section{Conclusion}

Understanding fundamental ethnic differences in IgAN has significant implications for both clinical care and future research. To date, many of the clinical trials of IgAN have been conducted in clinically homogenous, but histopathologically defined and ethnically restricted populations. The results of these studies might not necessarily be generalizable to other ethnic groups outside the study population. This is an important consideration when applying international treatment guidelines, such as the KDIGO guidelines, to the treatment of Chinese patients, and for the design of future studies. The identification of true differences in the pathogenic pathways of IgAN across different ethnic populations and from a Chinese perspective will influence treatment strategies across globally and eventually lead to targeted and personalized therapies.

\section{Conflict of Interest Statement}

The authors declare no conflicts of interest.
38

Glomerular Dis 2022;2:30-41 DOI: $10.1159 / 000520039$
Zhang/Zhang/Zhang 


\section{Funding Sources}

This study was funded by the National Natural Science Foundation of China (grant number 82070733), the Clinical Medicine Plus X - Young Scholars Project of Peking University (grant number PKU2021LCXQ017), the Fundamental Research Funds for the Central Universities, and CAMS Innovation Fund for Medical Sciences (grant number 2019-I2M-5-046), and "PRO-Run" Fund of the Nephrology Group of CEBM.

\section{Author Contributions}

Z. Zhang and Y.M. Zhang wrote and edited the manuscript; $\mathrm{H}$. Zhang conceived and edited the manuscript.

\section{References}

1 Wyatt RJ, Julian BA. IgA nephropathy. N Engl J Med. 2013;368(25):2402-14.

2 Barratt J, Feehally J. IgA nephropathy. J Am Soc Nephrol. 2005;16(7):2088-97.

3 D'Amico G. Natural history of idiopathic IgA nephropathy and factors predictive of disease outcome. Semin Nephrol. 2004;24(3):179-96.

4 Jennette JC. The immunohistology of IgA nephropathy. Am J Kidney Dis. 1988;12(5):34852.

5 Conley ME, Cooper MD, Michael AF. Selective deposition of immunoglobulin A1 in immunoglobulin A nephropathy, anaphylactoid purpura nephritis, and systemic lupus erythematosus. J Clin Invest. 1980;66(6):1432.

6 Allen AC, Bailey EM, Brenchley PE, Buck KS, Barratt J, Feehally J. Mesangial IgA1 in IgA nephropathy exhibits aberrant O-glycosylation: observations in three patients. Kidney Int. 2001;60(3):969-73.

7 Hiki Y, Odani H, Takahashi M, Yasuda Y, Nishimoto A, Iwase H, et al. Mass spectrometry proves under-O-glycosylation of glomerular IgA1 in IgA nephropathy. Kidney Int. 2001;59(3):1077-85.

8 Suzuki H, Kiryluk K, Novak J, Moldoveanu Z, Herr AB, Renfrow MB, et al. The pathophysiology of IgA nephropathy. J Am Soc Nephrol. 2011;22(10):1795-803.

9 Li L, Li LS, Chen HP, Zhou HZ, Ji DX, Tang $Z$, et al. Pathological classification and clinical characteristics of primary glomerulonephritis in China: bioptic study of 1001 cases. Zhonghua Yi Xue Za Zhi. 1989;69(1):20-3.

10 Gesualdo L, Di Palma AM, Morrone LF, Strippoli GF, Schena FP. The Italian experience of the national registry of renal biopsies. Kidney Int. 2004;66(3):890-4.

11 Hanko JB, Mullan RN, O'Rourke DM, McNamee PT, Maxwell AP, Courtney AE. The changing pattern of adult primary glomerular disease. Nephrol Dial Transplant. 2009; 24(10):3050-4.

12 Golay V, Trivedi M, Abraham A, Roychowdhary A, Pandey R. The spectrum of glomerular diseases in a single center: a clinicopathological correlation. Indian J Nephrol. 2013; 23(3): 168-75

13 Braun N, Schweisfurth A, Lohöfener C, Lange C, Gründemann C, Kundt G, et al. Epidemiology of glomerulonephritis in Northern Germany. Int Urol Nephrol. 2011;43(4):1117-26.
14 Browne O, Doyle GD, Campbell E. An immunohistological study of IgA nephropathy and a report of its incidence in Ireland. Ir J Med Sci. 1985; 154:461.

15 Mesquita M, Fosso C, Bakoto Sol E, Libertalis M, Corazza F, Vanden Houte K, et al. Renal biopsy findings in Belgium: a retrospective single center analysis. Acta Clin Belg. 2011; 66(2):104-9.

16 Rivera F, López-Gómez JM, Pérez-García R, Spanish Registry of G. Clinicopathologic correlations of renal pathology in Spain. Kidney Int. 2004;66(3):898-904.

17 Knoop T, Vikse BE, Svarstad E, Leh S, Reisæter AV, Bjørneklett R. Mortality in patients with IgA nephropathy. Am J Kidney Dis. 2013;62(5):883-90.

18 Lee H, Paik JH, Hyun JR, Kim DK, Chin HJ, $\mathrm{Oh} \mathrm{YK}$, et al. Long-term prognosis of clinically early IgA nephropathy is not always favorable. BMC Nephrology. 2014;15:94.

19 Feehally J, Cameron JS. IgA nephropathy: progress before and since Berger. Am J Kidney Dis. 2011;58(2):310-9.

20 Schena FP, Nistor I. Epidemiology of IgA nephropathy: a global perspective. Semin Nephrol. 2018;38(5):435-42.

21 Zhai Y, Xu H, Shen Q, Cao Q, Zhu G, Wei M, et al. Renal histological features of school-age children with asymptomatic haematuria and/ or proteinuria: a multicenter study. Nephrology. 2014;19(7):426-31.

22 Cho BS, Hahn WH, Cheong HI, Lim I, Ko CW, Kim SY, et al. A nationwide study of mass urine screening tests on Korean school children and implications for chronic kidney disease management. Clin Exp Nephrol. 2013;17(2):205-10.

23 Hu R, Quan S, Wang Y, Zhou Y, Zhang Y, Liu $\mathrm{L}$, et al. Spectrum of biopsy proven renal diseases in central China: a 10-year retrospective study based on 34,630 cases. Sci Rep. 2020; 10(1):10994.

24 Beukhof JR, Kardaun O, Schaafsma W, Poortema K, Donker AJ, Hoedemaeker PJ, et al. Toward individual prognosis of IgA nephropathy. Kidney Int. 1986;29(2):549-56.

25 Manno C, Strippoli GF, D'Altri C, Torres D, Rossini M, Schena FP. A novel simpler histological classification for renal survival in IgA nephropathy: a retrospective study. Am J Kidney Dis. 2007;49(6):763-75.
26 Yu X, Yang X. Peritoneal dialysis in China: meeting the challenge of chronic kidney failure. Am J Kidney Dis. 2015;65(1):147-51.

27 Allison SJ. Study warns of burgeoning health burden of CKD in China. Nat Rev Nephrol. 2012;8(5):251.

28 Kiryluk K, Li Y, Scolari F, Sanna-Cherchi S, Choi M, Verbitsky M, et al. Discovery of new risk loci for IgA nephropathy implicates genes involved in immunity against intestinal pathogens. Nat Genet. 2014;46(11): 1187-96.

29 Gharavi AG, Kiryluk K, Choi M, Li Y, Hou P, Xie J, et al. Genome-wide association study identifies susceptibility loci for IgA nephropathy. Nat Genet. 2011;43(4):321-7.

30 Feehally J, Farrall M, Boland A, Gale DP, Gut I, Heath S, et al. HLA has strongest association with IgA nephropathy in genome-wide analysis. J Am Soc Nephrol. 2010;21(10):1791-7.

31 Yu XQ, Li M, Zhang H, Low HQ, Wei X, Wang JQ, et al. A genome-wide association study in Han Chinese identifies multiple susceptibility loci for IgA nephropathy. Nat Genet. 2011;44(2):178-82.

32 Li M, Foo JN, Wang JQ, Low HQ, Tang XQ, Toh KY, et al. Identification of new susceptibility loci for IgA nephropathy in Han Chinese. Nat Commun. 2015;6:7270.

33 Li M, Wang L, Shi DC, Foo JN, Zhong Z, Khor CC, et al. Genome-wide meta-analysis identifies three novel susceptibility loci and reveals ethnic heterogeneity of genetic susceptibility for IgA nephropathy. J Am Soc Nephrol. 2020;31(12):2949-63.

34 John H, Stewart MREM, McDonald SP. Incidence of end-stage renal disease in overseasborn, compared with Australian-born, nonindigenous Australians. Nephrology. 2004; 9(4):247-52.

35 Hall YN, Fuentes EF, Chertow GM, Olson JL. Race/ethnicity and disease severity in IgA nephropathy. BMC Nephrol. 2004;5:10.

36 Zhang YM, Zhou XJ, Zhang H. What genetics tells us about the pathogenesis of IgA nephropathy: the role of immune factors and infection. Kidney Int Rep. 2017;2(3):318-31.

37 Zeng CH, Le W, Ni Z, Zhang M, Miao L, Luo $\mathrm{P}$, et al. A multicenter application and evaluation of the oxford classification of IgA nephropathy in adult chinese patients. Am J Kidney Dis. 2012;60(5):812-20. 
38 Coppo R, Troyanov S, Bellur S, Cattran D, Cook HT, Feehally J, et al. Validation of the Oxford classification of IgA nephropathy in cohorts with different presentations and treatments. Kidney Int. 2014;86(4):828-36.

39 Li M, Yu XQ. Genetic determinants of IgA nephropathy: eastern perspective. Semin Nephrol. 2018;38(5):455-60.

40 Endo Y, Hara M. Glomerular IgA deposition in pulmonary diseases. Kidney Int. 1986; 29(2):557-62.

41 Kano T, Suzuki H, Makita Y, Fukao Y, Suzuki Y. Nasal-associated lymphoid tissue is the major induction site for nephritogenic IgA in murine IgA nephropathy. Kidney Int. 2021; 100(2):364-76

42 Xie Y, Chen X, Nishi S, Narita I, Gejyo F. Relationship between tonsils and IgA nephropathy as well as indications of tonsillectomy. Kidney Int. 2004;65(4):1135-44.

43 Xie Y, Nishi S, Ueno M, Imai N, Sakatsume M, Narita I, et al. The efficacy of tonsillectomy on long-term renal survival in patients with IgA nephropathy. Kidney Int. 2003;63(5): $1861-7$.

44 Suzuki Y, Monteiro RC, Coppo R, Suzuki H. The phenotypic difference of IgA nephropathy and its race/gender-dependent molecular mechanisms. Kidney360. 2021;2(8):1339-48.

45 Fellström BC, Barratt J, Cook H, Coppo R, Feehally J, de Fijter JW, et al. Targeted-release budesonide versus placebo in patients with IgA nephropathy (NEFIGAN): a doubleblind, randomised, placebo-controlled phase 2b trial. Lancet. 2017;389(10084):2117-27.

46 Moldoveanu Z, Wyatt RJ, Lee JY, Tomana M, Julian BA, Mestecky J, et al. Patients with IgA nephropathy have increased serum galactosedeficient IgA1 levels. Kidney Int. 2007;71(11) 1148-54.

47 Berthoux F, Suzuki H, Thibaudin L, Yanagawa H, Maillard N, Mariat C, et al. Autoantibodies targeting galactose-deficient IgA1 associate with progression of IgA nephropathy. J Am Soc Nephrol. 2012;23(9):157987.

48 Shimozato S, Hiki Y, Odani H, Takahashi K, Yamamoto K, Sugiyama S. Serum under-galactosylated IgA1 is increased in Japanese patients with IgA nephropathy. Nephrol Dial Transplant. 2008;23(6):1931-9.

49 Zhao N, Hou P, Lv J, Moldoveanu Z, Li Y, Kiryluk K, et al. The level of galactose-deficient IgA1 in the sera of patients with IgA nephropathy is associated with disease progression. Kidney Int. 2012;82(7):790-6.

50 Hastings MC, Moldoveanu Z, Julian BA, Novak J, Sanders JT, McGlothan KR, et al. Galactose-deficient IgA1 in African Americans with IgA nephropathy: serum levels and heritability. Clin J Am Soc Nephrol. 2010;5(11): 2069-74.

51 Kiryluk K, Li Y, Moldoveanu Z, Suzuki H, Reily C, Hou P, et al. GWAS for serum galactose-deficient IgA1 implicates critical genes of the O-glycosylation pathway. PLoS Genet. 2017;13(2):e1006609.
52 Gale DP, Molyneux K, Wimbury D, Higgins $\mathrm{P}$, Levine AP, Caplin B, et al. Galactosylation of IgA1 is associated with common variation in C1GALT1. J Am Soc Nephrol. 2017;28(7): 2158-66.

53 Wang Y-N, Zhou X-J, Chen P, Yu G-Z, Zhang $\mathrm{X}, \mathrm{Hou} \mathrm{P}$, et al. Interaction between GALNT12 and C1GALT1 associates with galactose-deficient IgA1 and IgA nephropathy. JASN. 2021; 32(3):545-52.

54 Yeo SC, Goh SM, Barratt J. Is immunoglobulin A nephropathy different in different ethnic populations? Nephrology. 2019;24(9): 885-95.

55 Novak J, Barratt J, Julian BA, Renfrow MB Aberrant glycosylation of the IgA1 molecule in IgA nephropathy. Semin Nephrol. 2018; 38(5):461-76

56 Takahashi K, Smith AD, Poulsen K, Kilian M, Julian BA, Mestecky J, et al. Naturally occurring structural isomers in serum IgA1 o-glycosylation. J Proteome Res. 2012;11(2):692-702.

57 Katafuchi R, Nagae H, Masutani K, Tsuruya K, Mitsuiki K. Comprehensive evaluation of the significance of immunofluorescent findings on clinicopathological features in IgA nephropathy. Clin Exp Nephrol. 2019;23(2): 169-81.

58 Kim SJ, Koo HM, Lim BJ, Oh HJ, Yoo DE, Shin DH, et al. Decreased circulating C3 levels and mesangial C3 deposition predict renal outcome in patients with IgA nephropathy. PLoS One. 2012;7(7):e40495.

59 Paunas TIF, Finne K, Leh S, Marti HP, Mollnes TE, Berven F, et al. Glomerular abundance of complement proteins characterized by proteomic analysis of laser-captured microdissected glomeruli associates with progressive disease in IgA nephropathy. Clin Proteomics. 2017;14:30.

60 Zhang JJ, Jiang L, Liu G, Wang SX, Zou WZ, Zhang $\mathrm{H}$, et al. Levels of urinary complement factor $\mathrm{H}$ in patients with IgA nephropathy are closely associated with disease activity. Scand J Immunol. 2009;69(5):457-64.

61 Zhu L, Guo WY, Shi SF, Liu LJ, Lv JC, Medjeral-Thomas NR, et al. Circulating complement factor $\mathrm{H}$-related protein 5 levels contribute to development and progression of $\operatorname{IgA}$ nephropathy. Kidney Int. 2018;94(1):150-8.

62 Xie J, Kiryluk K, Li Y, Mladkova N, Zhu L, Hou $\mathrm{P}$, et al. Fine mapping implicates a deletion of CFHR1 and CFHR3 in protection from IgA nephropathy in Han Chinese. J Am Soc Nephrol. 2016;27(10):3187-94.

63 Roos A, Rastaldi MP, Calvaresi N, Oortwijn $\mathrm{BD}$, Schlagwein N, van Gijlswijk-Janssen DJ, et al. Glomerular activation of the lectin pathway of complement in IgA nephropathy is associated with more severe renal disease. J Am Soc Nephrol. 2006;17(6):1724-34.

64 Liu Z, Xu B, Nameta M, Zhang Y, Magdeldin $S$, Yoshida Y, et al. Profiling of kidney vascular endothelial cell plasma membrane proteins by liquid chromatography-tandem mass spectrometry. Clin Exp Nephrol. 2012;17(3): 327-37.
65 Liu LL, Jiang Y, Wang LN, Liu N. Urinary mannose-binding lectin is a biomarker for predicting the progression of immunoglobulin (Ig)A nephropathy. Clin Exp Immunol. 2012;169(2):148-55.

66 Guo WY, Zhu L, Meng SJ, Shi SF, Liu LJ, Lv JC, et al. Mannose-binding lectin levels could predict prognosis in IgA nephropathy. J Am Soc Nephrol. 2017;28(11):3175-81.

67 Lv J, Zhang H, Zhou Y, Li G, Zou W, Wang $H$. Natural history of immunoglobulin A nephropathy and predictive factors of prognosis: a long-term follow up of 204 cases in China. Nephrology. 2008;13(3):242-6.

68 Li X, Liu Y, Lv J, Shi S, Liu L, Chen Y, et al. Progression of IgA nephropathy under current therapy regimen in a Chinese population. Clin J Am Soc Nephrol. 2014;9(3):484-9.

69 Working Group of the International Ig ANN, the Renal Pathology Society; Cattran DC, Cattran DC, Coppo R, Cook HT, Feehally J, et al. The Oxford classification of IgA nephropathy: rationale, clinicopathological correlations, and classification. Kidney Int. 2009; 76(5):534-45.

70 Goto M, Wakai K, Kawamura T, Ando M, Endoh M, Tomino Y. A scoring system to predict renal outcome in IgA nephropathy: a nationwide 10-year prospective cohort study. Nephrol Dial Transplant. 2009;24(10):306874.

71 Goto M, Kawamura T, Wakai K, Ando M, Endoh M, Tomino Y. Risk stratification for progression of IgA nephropathy using a decision tree induction algorithm. Nephrol Dial Transplant. 2009;24(4):1242-7.

72 Chen T, Li X, Li Y, Xia E, Qin Y, Liang S, et al. Prediction and risk stratification of kidney outcomes in IgA nephropathy. Am J Kidney Dis. 2019;74(3):300-9.

73 Jian Liu SD, Chen P, Cai G, Wang Y, Tang L, Liu S, et al. Development and validation of a prognostic nomogram for IgA nephropathy. Oncotarget. 2017;8(55):94371-81.

74 Berthoux F, Mohey H, Laurent B, Mariat C, Afiani A, Thibaudin L. Predicting the risk for dialysis or death in IgA nephropathy. J Am Soc Nephrol. 2011;22(4):752-61.

75 Barbour SJ, Coppo R, Zhang H, Liu ZH, Suzuki Y, Matsuzaki K, et al. Evaluating a new international risk-prediction tool in IgA nephropathy. JAMA Intern Med. 2019;179(7): 942-52.

76 Barbour SJ, Canney M, Coppo R, Zhang H, Liu ZH, Suzuki Y, et al. Improving treatment decisions using personalized risk assessment from the International IgA nephropathy prediction tool. Kidney Int. 2020;98(4):1009-19.

77 Barbour SJ, Coppo R, Er L, Russo ML, Liu ZH, Ding J, et al. Updating the international IgA nephropathy prediction tool for use in children. Kidney Int. 2021;99(6):1439-50.

78 Zhang Y, Guo L, Wang Z, Wang J, Er L, Barbour SJ, et al. External validation of international risk-prediction models of IgA nephropathy in an Asian-Caucasian cohort. Kidney Int Rep. 2020;5(10):1753-63. 
79 Schena FP, Anelli VW, Trotta J, Di Noia T, Manno C, Tripepi G, et al. Development and testing of an artificial intelligence tool for predicting end-stage kidney disease in patients with immunoglobulin A nephropathy. Kidney Int. 2021;99(5):1179-88.

80 Berthoux F, Suzuki H, Thibaudin L, Yanagawa $\mathrm{H}$, Maillard N, Mariat C, et al. Autoantibodies targeting galactose-deficient IgA1 associate with progression of IgA nephropathy. J Am Soc Nephrol. 2012;23(9):1579-87.

$81 \mathrm{Lv}$ J, Zhang H, Wong MG, Jardine MJ, Hladunewich $M$, Jha $V$, et al. Effect of oral methylprednisolone on clinical outcomes in patients with IgA nephropathy: the TESTING randomized clinical trial. JAMA. 2017;318(5): $432-42$.
82 Rauen T, Eitner F, Fitzner C, Sommerer C, Zeier M, Otte B, et al. Intensive Supportive Care plus Immunosuppression in IgA Nephropathy. N Engl J Med. 2015;373(23): 2225-36.

83 Hou JH, Le WB, Chen N, Wang WM, Liu ZS, Liu D, et al. Mycophenolate mofetil combined with prednisone versus full-dose prednisone in IgA nephropathy with active proliferative lesions: a randomized controlled trial. Am J Kidney Dis. 2017;69(6):788-95.

84 Schubart A, Anderson K, Mainolfi N, Sellner $\mathrm{H}$, Ehara T, Adams CM, et al. Small-molecule factor B inhibitor for the treatment of complement-mediated diseases. Proc Natl Acad Sci USA. 2019;116(16):7926-31.
85 Lafayette RA, Rovin BH, Reich HN, Tumlin JA, Floege J, Barratt J. Safety, tolerability and efficacy of narsoplimab, a novel MASP-2 inhibitor for the treatment of IgA nephropathy. Kidney Int Rep. 2020;5(11):2032-41.

86 Bjorneklett R, Vikse BE, Bostad L, Leivestad $\mathrm{T}$, Iversen BM. Long-term risk of ESRD in IgAN; validation of Japanese prognostic model in a Norwegian cohort. Nephrol Dial Transplant. 2012;27(4):1485-91.

87 Knoop T, Vågane AM, Vikse BE, Svarstad E, Magnúsdóttir BT, Leh S, et al. Addition of eGFR and Age improves the prognostic absolute renal risk-model in 1,134 Norwegian patients with IgA nephropathy. Am J Nephrol. 2015;41(3):210-9.

88 Xie J, Lv J, Wang W, Li G, Liu Z, Chen H, et al. Kidney failure risk prediction equations in Iga nephropathy: a multicenter risk assessment study in Chinese Patients. Am J Kidney Dis. 2018;72(3):371-80. 